\title{
Infant Oral Health: Focus on Caries Prevention during Pregnancy
}

\author{
Marcelle M Nascimento* \\ Associate Professor, Department of Restorative Dental Sciences, Division of Operative Dentistry, College of Dentistry, University of Florida, Gaines- \\ ville, FL, USA
}

${ }^{\star}$ Corresponding author: Marcelle M Nascimento, Associate Professor, Department of Restorative Dental Sciences, Division of Operative Dentistry, College of Dentistry, University of Florida, Gainesville, FL, USA

Received: May 05, 2021; Accepted: May 14, 2021; Published: May 27, 2021

\begin{abstract}
Tooth decay or dental caries is the most common chronic disease of childhood. However, caries is also highly preventable through a combination of oral hygiene, the use of fluoride and dietary measures. Prevention of caries should start in the prenatal period because the maternal caries status is strongly associated with the caries status of their children. This short review will address the importance of early risk assessment for identification of parentinfant groups who are at high risk for Early Childhood Caries (ECC) and would benefit significantly from early preventive intervention.
\end{abstract}

Keywords: Dental caries, Early childhood caries, Pregnancy, Infant oral health

\section{Introduction}

Tooth decay or dental caries continues to be a major public health problem worldwide. Caries affects nearly $23 \%$ of U.S. children aged 2-5 years and $56 \%$ of those aged 6-8 years [1]. Early childhood caries (ECC) can have life-long consequences on the overall health of children due to severe pain, abscesses, chewing difficulty, malnutrition, poor speech articulation, low self-esteem, and increased risk for future caries [2,3]. Importantly, pregnant women are at higher caries risk due to reasons such as increased acidity in the oral cavity, sugary dietary cravings, and limited attention to oral health [4]. The lack of maternal dental care and the transmission of caries pathogens from caries-active mothers to infants lead to increased risk for childhood caries. Appropriate dental care and caries prevention during pregnancy may decrease infant and childhood caries. Therefore, early risk assessment is critical- for identification of parent-infant groups who are at high risk for ECC and would benefit significantly from early preventive intervention.

\section{The Caries Process}

The term caries lesions are the scientific term used to describe the signs and symptoms of the disease of dental caries (or tooth decay). A caries lesion is the result of a localized destruction (or demineralization) of the tooth surface caused by acids produced from the fermentation of dietary carbohydrates (mainly free sugars) by dental plaque bacteria. The destruction can affect tooth enamel, dentin and cementum. The first sign of a caries lesion on enamel that can be detected with the naked eye is called a white spot lesion. The caries process can be stopped or reversed at this point because enamel can repair itself by using minerals from saliva, and fluoride from toothpaste or other sources. But if the caries process continues, more minerals will be lost. Over time, the enamel is weakened and destroyed, forming a cavity. A cavity is a permanent damage on the tooth that a dentist has to repair with a filling. Unquestionably, a combination of oral hygiene, the use of fluoride and dietary measures must take place to avoid the onset of white spot lesions and their consequent progression into cavities.

\section{Caries Prevention}

Preventing caries and promoting oral health begins with establishing healthy habits in infancy. Dental caries is preventable through a combination of three major behaviors that are proven effective: 1) oral hygiene methods, 2) frequent exposure to fluoride, and 3) a diet that limits the intake of sugary components [5]. Oral Hygiene $(\mathrm{OH})$ is key to remove the acid-producing dental plaque that can demineralize the teeth. The best $\mathrm{OH}$ recommendation includes tooth brushing twice a day (especially at bedtime) followed by flossing. In addition, fluoride is a mineral that can prevent, stop the progression, and even reverse early white spot lesions. Fluoride prevents mineral loss in tooth enamel and replaces lost minerals. Sources of fluoride include: drinking fluoridated water from a community water supply (about $74 \%$ of Americans served by a community water supply system receive fluoridated water, brushing teeth with a fluoride toothpaste, rinsing your mouth daily with a fluoride mouth rinse, and application of a fluoride gel or varnish to tooth surfaces at dental clinics by dental professionals. Also extremely important in caries prevention is a diet low in foods and drinks containing sugar and starches, which can be metabolized into acids by caries pathogens.

\section{Infant Oral Health}

The American Academy of Pediatric Dentistry (AAPD) recognizes infant oral health care as a foundation for offering preventive education and dental care that can enhance the opportunity for a lifetime free 
from preventable oral diseases [3]. The AAPD recommends that every infant receive an oral health risk assessment from his/her primary health care provider or qualified health care professional by 6 months of age [3], and that parents establish a dental home for infants by 12 months of age [6]. Caries risk assessment for infants allows for the implementation of appropriate prevention and management strategies as the primary dentition begins to erupt [3].

This initial oral health risk assessment should include: 1) evaluation of medical (infant) and dental (parent and infant) histories; 2) a thorough oral examination; 3 ) an assessment of the patient's risk of developing oral diseases of soft and hard tissues; 4) education on infant oral health, oral hygiene measures (such as age-appropriate tooth brushing demonstration), nonnutritive sucking habits, teething, injury prevention, and the dietary effects on the dentition; 5) anticipatory guidance regarding dental and oral development; 6) evaluation and optimization of fluoride exposure; 7) development of a preventive treatment plan that includes prophylaxis, fluoride varnish and periodic re-assessment appointments; and 8) referral to the appropriate health professional if specialized intervention is necessary.

The ultimate goal of early assessment is the timely delivery of educational information to populations at high risk for developing caries and prevention of later surgical intervention. Despite the importance of early risk assessment, there is an unfortunate lack of access to dental services among young children in the United States, coupled with a limited workforce to address their oral health needs. In fact, the implementation of Infant Oral Health Programs (IOHP) in dental schools is not only an important community resource for preventive dental care for young children at high risk for caries but should increase the workforce providing care to this population [7].

\section{References}

1. Dye BA, Thornton-Evans G, Li X, Iafolla TJ (2015) Dental caries and sealant prevalence in children and adolescents in the United States, 2011-2012. NCHS Data Brief 1-8. [crossref]

2. Ramos-Gomez FJ (2005) Clinical considerations for an infant oral health care program. Compend Contin Educ Dent 26: 17-23. [crossref]

3. Dentistry AAoP (2014) Guideline on Infant Oral Health Care. Pediatr Dent 36: 1415. [crossref]

4. Silk H, Douglass AB, Douglass JM, Silk L (2008) Oral health during pregnancy. Am Fam Physician 77: 1139-1144.

5. Kidd E, Fejerskov O (2013) Changing concepts in cariology: forty years on. Dent Update 40: 277-278, 80-82, 85-86. [crossref]

6. Dentistry AAoP (2011) Policy on the dental home. Pediatr Dent 33: 24-25. [crossref]

7. Nascimento MM, Mugayar L, Tomar SL, Garvan CW, Catalanotto FA, et al. (2016) The Impact of an Infant Oral Health Program on Dental Students' Knowledge and Attitudes. J Dent Educ 80: 1328-1336. [crossref]

\section{Citation:}

